(C) [2007] IEEE. Reprinted, with permission, from [Teddy M. Cheng, Andrey V. Savkin, Branko G. Celler, Lu Wang, Steven W. Su, A nonlinear dynamic model for heart rate response to treadmill walking exercise, Engineering in Medicine and Biology Society, 2007. EMBS 2007. 29th Annual International Conference of the IEEE, 22-26 Aug. 2007]. This material is posted here with permission of the IEEE. Such permission of the IEEE does not in any way imply IEEE endorsement of any of the University of Technology, Sydney's products or services. Internal or personal use of this material is permitted. However, permission to reprint/republish this material for advertising or promotional purposes or for creating new collective works for resale or redistribution must be obtained from the IEEE by writing to pubs-permissions@ieee.org. By choosing to view this document, you agree to all provisions of the copyright laws protecting it 


\title{
A nonlinear dynamic model for heart rate response to treadmill walking exercise
}

\author{
Teddy M. Cheng, Andrey V. Savkin, Branko G. Celler, Lu Wang, Steven W. Su
}

\begin{abstract}
A dynamic model of the heart rate response to treadmill walking exercise is presented. The model is a feedback interconnected system; the subsystem in the forward path represents the neural response to exercise, while the subsystem in the feedback path describes the peripheral local response. The parameters of the model were estimated from 5 healthy adult male subjects, each undertaking 3 sets of walking exercise at different speeds. Simulated responses from the model closely match the experimental data both in the exercise and the recovery phases. The model will be useful in explaining the cardiovascular response to exercise and in the design of exercise protocols for individuals.
\end{abstract}

\section{INTRODUCTION}

Metabolic demand increases during dynamic exercise. In response to this, the cardiovascular system increases the delivery of blood and oxygen to working muscles. This is reflected by an increase in heart rate and stroke volume. Among these two physiological variables, heart rate is relatively easier and cheaper to measure. Monitoring heart rate response then provides a convenient way of estimating oxygen consumption and energy expenditure during exercise. Therefore, if the heart rate response is modelled, our understanding of exercise physiology will improve. This may also lead to improved training protocols for athletics and the assessment of physical fitness and health the individual [1]. Understanding the aetiology of heart rate response during, and recovery after, an exercise, may also be beneficial to predicting cardiovascular disease mortality [2] [3].

Broden et al. [4] and Hajek et al. [5] modelled the heart rate response, both during exercise and recovery, from a regulation point of view. Namely, for each work load, they assumed that there was a target heart rate corresponding to the work load and the job of the autonomic nervous system was to regulate this particular heart rate. No local responses, such as effects from local metabolites and/or the increase in body temperature, were considered in their models. If the exercise duration was short, less than six minutes in their studies, the target heart rate would not drift significantly and their models were reliable. However, as shown in, e.g. [6][8], heart rate will continue to increase during prolonged exercise.

In this paper, we propose a dynamic model that describes the heart rate response to treadmill walking exercise both

This work was supported by the Australian Research Council.

Teddy M. Cheng, Andrey V. Savkin, Branko G. Celler, and Lu Wang are with the School of Electrical Engineering and Telecommunications, the University of New South Wales, Sydney, NSW 2052, Australia.

Steven W. Su is with the Faculty of Engineering, the University of Technology, Sydney, NSW 2007, Australia

Corresponding author: Teddy M. Cheng (email: t.cheng@ieee.org) in the exercising and the recovery phases. Using a different approach than [4] and [5], we model the heart rate response from the neural and the local responses perspective. The advantage of this approach is that the model can describe the heart rate response over a longer exercise duration.

\section{The Proposed Model}

In this paper, we propose the following nonlinear control systems to model the heart rate response to treadmill walking exercise:

$$
\begin{aligned}
\dot{x}_{1}(t) & =-a_{1} x_{1}(t)+x_{2}(t)+g(u(t)) \\
\dot{x}_{2}(t) & =-a_{4}\left(x_{2}(t)-\tanh \left(x_{2}(t)\right)\right)+a_{5} x_{1}(t) \\
y(t) & =x_{1}(t)
\end{aligned}
$$

where

$g(u(t)):=\frac{a_{2} u^{2}(t)}{1+\exp \left(-u(t)+a_{3}\right)}, u(t):= \begin{cases}v & \text { for } t \leq t_{s} \\ 0 & \text { for } t>t_{s}\end{cases}$

and $x(0)=\left[\begin{array}{ll}x_{1}(0) & x_{2}(0)\end{array}\right]^{T}=0, y(t)=\Delta H R(t)=$ $H R(t)-H R_{\text {rest }}$, namely, the change in heart rate from rest, and $a_{1}, \ldots, a_{5}$ are positive scalars. The time $t_{s}$ is the time when a subject stops exercising. The scalar $v$ in the control input $u(t)$ represents the speed of the treadmill. System (1) can be viewed as a feedback interconnected system. The component $x_{1}(t)$ can be interpreted as the change of heart rate $(\Delta \mathrm{bpm})$ due to the neural response to exercise, including both the parasympathetic and the sympathetic neural inputs. It is understood that for a low intensity exercise, the increase in heart rate is mainly due to the vagal withdrawal. Whereas for a higher intensity exercise, the increase in heart rate is dominated by the sympathetic activation, see e.g. [6] and [9].

The component $x_{2}$ is utilised in describing the complex slow-acting effects from, e.g. the hormonal systems, the peripheral local metabolism, the increase in body temperature, and/or the loss of body fluid due to sweating and hyperventilation, etc.. In the case of the peripheral local metabolism, the metabolic byproducts, such as adenosine, $\mathrm{K}^{+}, \mathrm{H}^{+}, \mathrm{PO}_{4}^{3-}$, lactic acid and other metabolites, cause vasodilatation and hyperaemia in active muscles (see, e.g. [9]).

Vasodilatation in the active muscles cause a reduction in total peripheral resistance which in turn causes a drop in mean arterial pressure. In order to maintain the arterial pressure, the cardiac output needs to be increased, meaning that stroke volume and heart rate have to be increased via the baroreceptor reflex. So, the feedback signal $x_{2}$, which can be thought of as a dynamic disturbance input to the $x_{1}$ 


\begin{tabular}{|c|c|c|c|c|}
\hline Subject & Age & Height $(\mathrm{cm})$ & Weight $(\mathrm{kg})$ & BMI \\
\hline 1 & 25 & 176 & 78 & 25 \\
\hline 2 & 28 & 171 & 59 & 20 \\
\hline 3 & 27 & 174 & 53 & 18 \\
\hline 4 & 23 & 176 & 85 & 27 \\
\hline 5 & 38 & 178 & 75 & 24 \\
\hline
\end{tabular}

TABLE I

PhySiCAL CHARACTERISTICS OF THE SUBJECTS: AGE, HEIGHT, WEIGHT, AND BMI (BODY MASS INDEX)

subsystem, is a reaction to the effects from the peripheral local metabolism. In other words, the metabolites from the peripheral local metabolism accelerate the heart rate during exercise [10]. Since $x_{2}$ is a nonlinear function of $x_{1}$ and therefore has the same unit as $x_{1}$ (i.e. $\Delta$ bpm).

The nonlinear term $\left(x_{2}(t)-\tanh \left(x_{2}(t)\right)\right)$ in $\dot{x}_{2}(t)$ is to model the slow recovery of heart rate after exercise. The term $\left(x_{2}(\cdot)-\tanh (\cdot)\right)$ is used due to its zero gradient at the origin, causing the solution of the $x_{2}(t)$ subsystem converging to the origin $x_{2}=0$ much slower than that of a linear system in the neighbourhood of the origin.

The function $g(u)$ explains the nonlinear increase of the heart rate in response to the increase in walking speed. We choose $g(u)$ in this particular form because it vanishes at zero, i.e., $g(0)=0$, and there is a curvilinear relationship between aerobic demand and walking speed (see, e.g. [11] and [10]). Furthermore, as observed in [12], there is a sharp increase in the heart rate when a person walks beyond a certain speed.

\section{EXPERIMENTAL VERIFICATION}

\section{A. Subjects}

Five healthy male subjects were studied. The physical characteristics of the subjects are given in Table I.

\section{B. Procedure}

Each subject completed three exercise sessions in separate occasions. In each session, a subject was requested to walk on a treadmill at a given speed $(5 \mathrm{~km} / \mathrm{h}, 6 \mathrm{~km} / \mathrm{h}$, and $7 \mathrm{~km} / \mathrm{h})$ for 15 minutes with a recovery period of 20 minutes. After three sessions, each subject completed the treadmill walking exercise at the three different speeds. The heart rate of the subjects were recorded from at least 3 minutes before the starting until 20 minutes after the cessation of the walking exercise. The period before the exercise and the 20-minute period after the exercise are the resting and recovery periods, respectively. During both the resting and the recovery periods, the subjects were requested to maintain a standing posture.

\section{Data acquisition and pre-processing}

In this study, the Powerjog " $G$ " series fully motorised medical grade treadmill was used. The heart rate of the subjects was monitored by the wireless Polar system and recorded by LABVIEW. The polar system generated pulses which were used to extract R-R intervals and, in turn, the heart rate. Due to electromagnetic interferences from some unknown external sources and the movements of the subjects, outliers were observed in the measured heart rate data. The outliers were removed so that the heart rate data were within a reasonable range. Then, the heart rate data were filtered using the moving average with a 5-second window.

Since the proposed system (1) is to model the change of heart rate from the resting situation, the resting heart rate for each subject at each intensity of walking exercise was estimated from the 3-minute resting period. The mean heart rate in this resting period was defined as the resting heart rate. Then all the heart rate data were subtracted by their respective resting heart rate.

\section{Parameter Estimation}

Using the measured heart rate data, the parameters in system (1) were estimated for each subject by the LevenbergMarquardt method. Since there were three sets of inputoutput measurements for each subject (where the input is the speed of the treadmill and the output is the heart rate), we estimated the parameters as if a multi-input multi-output system. In other words, we considered it as a parameter estimation problem of the following system:

$$
\dot{\mathbf{x}}(t)=\mathbf{f}(\mathbf{x}(t), a, u(t)), \quad y(t)=C \mathbf{x}(t)
$$

where $\mathbf{x}(0)=0, C=\left[\begin{array}{llllll}1 & 0 & 0 & 0 & 0 & 0 \\ 0 & 0 & 1 & 0 & 0 & 0 \\ 0 & 0 & 0 & 0 & 1 & 0\end{array}\right], \mathbf{x}=$ $\left[\begin{array}{lll}\mathbf{x}_{\mathbf{1}} & \mathbf{x}_{\mathbf{2}} & \mathbf{x}_{\mathbf{3}}\end{array}\right]^{T} \in \mathbb{R}^{6}, a=\left[\begin{array}{ll}a_{1} & a_{2} \ldots a_{5}\end{array}\right]^{T} \in \mathbb{R}^{5}, u=$ $\left[\begin{array}{lll}u_{1} & u_{2} & u_{3}\end{array}\right]^{T} \in \mathbb{R}^{3}$ and $y=\left[\begin{array}{lll}y_{1} & y_{2} & y_{3}\end{array}\right]^{T} \in \mathbb{R}^{3}$. For $i=1,2,3$, the vector $\mathbf{x}_{\mathbf{i}}:=\left[\begin{array}{ll}x_{i, 1} & x_{i, 2}\end{array}\right]^{T}$ and $y_{i}$ are the state vector and the output from the input $u_{i}$.

The objective function was $S(a)=\sum_{i=1}^{N}\left(y\left(t_{i}\right)-\right.$ $\left.\hat{y}\left(t_{i}, a\right)\right)^{T}\left(y\left(t_{i}\right)-\hat{y}\left(t_{i}, a\right)\right)$ where, for $i=1,2, \ldots, N, y\left(t_{i}\right)$ is the measurement of the output vector at time $t_{i}$ and $\hat{y}\left(t_{i}, a\right)$ is the output of system (3) with the parameter vector $a$. With the objective function $S(a)$, the Levenberg-Marquardt method was used to determine an estimate of $a$ which was denoted as $\hat{a}:=\left[\begin{array}{lll}\hat{a}_{1} & \hat{a}_{2} \ldots \hat{a}_{5}\end{array}\right]^{T}$ (see, e.g. [13], [14]).

For each subject, we assumed that the errors in the measurements for all the experiments were statistically independent and Gaussian distributed with a constant variance $\sigma_{e}^{2}$. Based on a linear approximate method (see e.g. [14]), an approximate $100(1-\alpha) \%$ independent confidence interval for each estimate was given by $\left(\hat{a}_{i}-\delta a_{i}, \hat{a}_{i}+\delta a_{i}\right)$, for $i=1,2, \ldots, 5$, with $\delta a_{i}=$ $\sqrt{\frac{p}{N m-p} S(\hat{a}) \mathcal{F}_{\alpha}(p, N m-p)\left[A^{-1}\right]_{i, i}}$, where $\mathcal{F}_{\alpha}(p, N m-$ $p$ ) denotes the upper $\alpha$ quantile for Fishers $\mathcal{F}$-distribution with $p$ and $N m-p$ degrees of freedom and the scalar $\left[A^{-1}\right]_{i, i}$ is the $(i, i)$ diagonal element of the inverse matrix $A$ that is defined as $A:=\sum_{i=1}^{N} G^{T}\left(t_{i}\right) C^{T} C G\left(t_{i}\right), G\left(t_{i}\right):=$ $\partial \mathbf{f} /\left.\partial a\right|_{a=\hat{a}, t=t_{i}}$. In this study, $N=2100, m=3$ and $p=5$. We then checked the adequacy of the model. First, for each subject, the variance of the errors in the measurements $\sigma_{e}^{2}$ was estimated by using the maximum value of the standard derivations of the heart rate during the resting periods in 
the three different experiments. The estimated value of $\sigma_{e}^{2}$ was denoted as $\hat{\sigma}_{e}^{2}$. If $S(\hat{a}) /\left(\hat{\sigma}_{e}^{2}(N m-p)\right)>\mathcal{F}_{\alpha}(N m-$ $p, q=180)$, it would imply that the model was not adequate (see [13]). An $\alpha$ level of 0.05 was used for obtaining the confidence intervals of parameter estimates and for all significance tests.

\section{RESUlTS}

Table II summaries the means and the standard deviations of the subjects' resting heart rate before each exercise session. The table also shows the estimated variances of the measurement error for the individual subject, and the test statistics for the model adequacy test are listed on the last column. All the test statistics are less than the critical value, indicating that the model is adequate for the experimental data recorded from all the subjects. Table III summaries the estimated parameters. The simulated heart rate responses with the proposed model for all the subjects are shown in Figures 1-5. All the simulated responses are consistent with the experimental data.

\begin{tabular}{|c|c|c|c|c|c|}
\hline \multirow{5}{*}{ Subject } & $\begin{array}{c}\mathrm{HR}_{\text {rest }} \\
5 \mathrm{~km} / \mathrm{h}\end{array}$ & $\begin{array}{c}\mathrm{HR}_{\text {rest }} \\
6 \mathrm{~km} / \mathrm{h}\end{array}$ & $\begin{array}{c}\mathrm{HR}_{\text {rest }} \\
7 \mathrm{~km} / \mathrm{h}\end{array}$ & \multirow{3}{*}{} & \multirow{2}{*}{$S(\hat{a})$} \\
\cline { 2 - 5 } & mean & mean & mean & $\hat{\sigma}_{e}^{2}$ & $\frac{S}{\hat{\sigma}_{e}^{2}(N m-p)}$ \\
& $(\mathrm{SD})$ & $(\mathrm{SD})$ & $(\mathrm{SD})$ & & \\
\hline 1 & 78.7 & 77.5 & 74.8 & 12.3 & 1.1 \\
& $(3.5)$ & $(3.0)$ & $(3.4)$ & & \\
\hline 2 & 61.9 & 60.3 & 57.6 & 17.1 & 1.0 \\
& $(3.9)$ & $(3.3)$ & $(4.13)$ & & \\
\hline 3 & 73.4 & 76.9 & 67.0 & 17.6 & 1.1 \\
& $(3.2)$ & $(2.9)$ & $(4.2)$ & & \\
\hline 4 & 85.1 & 80.8 & 76.5 & 26.0 & 0.8 \\
& $(4.1)$ & $(5.1)$ & $(4.3)$ & & \\
\hline 5 & 78.7 & 75.7 & 76.8 & 28.1 & 0.7 \\
& $(2.8)$ & $(3.3)$ & $(5.3)$ & & \\
\hline
\end{tabular}

TABLE II

MEANS AND STANDARd DERIVATIONS OF RESTING heART Rate, $\hat{\sigma}_{e}$, AND $\frac{S(\hat{a})}{\hat{\sigma}_{e}^{2}(N m-p)}$ FOR 5 DIFFERENT SUBJECTS.

\begin{tabular}{|c|c|c|c|c|c|}
\hline \multirow{2}{*}{ Subject } & \multicolumn{5}{|c|}{$\begin{array}{c}\text { Parameter estimates } \\
\text { Confidence intervals, } \delta a \text { ) }\end{array}$} \\
\cline { 2 - 6 } & $a_{1}$ & $a_{2}$ & $a_{3}$ & $a_{4}$ & $a_{5}$ \\
\hline 1 & 2.103 & 1.964 & 1.717 & 0.028 & 0.038 \\
& $(0.131)$ & $(0.114)$ & $(0.556)$ & $(0.006)$ & $(0.005)$ \\
\hline 2 & 1.844 & 2.747 & 5.263 & 0.099 & 0.068 \\
& $(0.116)$ & $(0.152)$ & $(0.048)$ & $(0.008)$ & $(0.009)$ \\
\hline 3 & 1.833 & 1.688 & 4.686 & 0.057 & 0.065 \\
& $(0.168)$ & $(0.139)$ & $(0.082)$ & $(0.007)$ & $(0.010)$ \\
\hline 4 & 1.804 & 1.212 & 4.469 & 0.210 & 0.164 \\
& $(0.282)$ & $(0.150)$ & $(0.114)$ & $(0.025)$ & $(0.048)$ \\
\hline 5 & 2.592 & 2.109 & 4.684 & 0.074 & 0.032 \\
& $(0.299)$ & $(0.226)$ & $(0.112)$ & $(0.030)$ & $(0.013)$ \\
\hline
\end{tabular}

TABLE III

ESTIMATED PARAMETER VALUES FOR 5 DIFFERENT SUBJECTS.

\section{CONCLUding REMARKS AND DiscusSiONS}

In this study, a dynamic model describing the heart rate response to the treadmill walking exercise was proposed. The

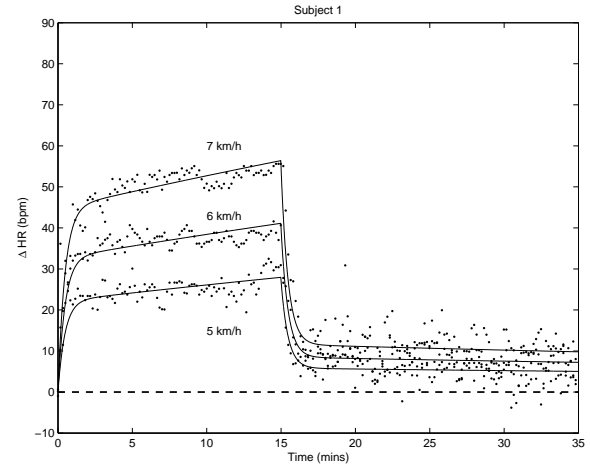

Fig. 1. Heart rate responses of Subject 1: actual responses (dots), simulated responses (solid lines)

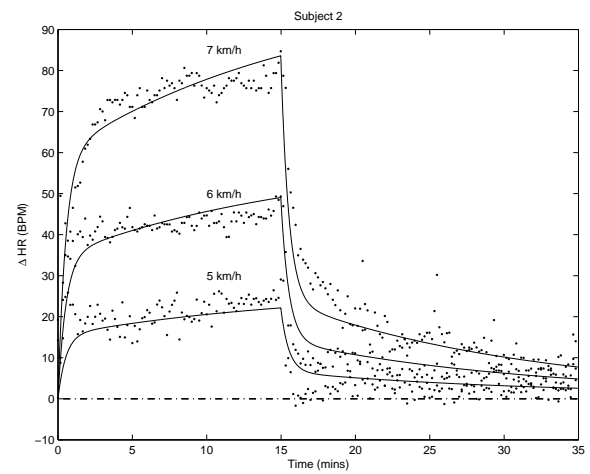

Fig. 2. Heart rate responses of Subject 2: actual responses (dots), simulated responses (solid lines)

simulation results of the model were found to be consistent with the experimental data. Also, the structure of the model could be explained from a physiological point of view. The proposed model is a feedback interconnected system. It contains a fast-response subsystem in the forward path that can be used to describe the neural response. Whereas, the feedback subsystem can be utilised to describe the slow peripheral local response. Therefore, the model would be useful in describing the heart rate response due to both the neural and the local responses to exercise.

A limitation of the proposed model is that it cannot describe the switching effects between vagal and sympathetic activities during and after exercise. For example, when a person performing a high intensity exercise, the increase in heart rate is due to both the parasympathetic and the sympathetic effects. However, the parasympathetic effect can only drive the heart rate up to a certain point. As shown in reference [6], the increase of heart rate above $\approx 100$ beats/min is mainly controlled by the sympathetic activities. It has also been observed that the response times for the parasympathetic and the sympathetic activities are different, e.g. the rate of heart rate increase is much faster in vagal withdrawal than that of sympathetic activation (see e.g. [6]). Therefore, in order to model the above-mentioned behaviour properly, the parameter $a_{1}$ should depend on the state $x_{1}(t)$. 


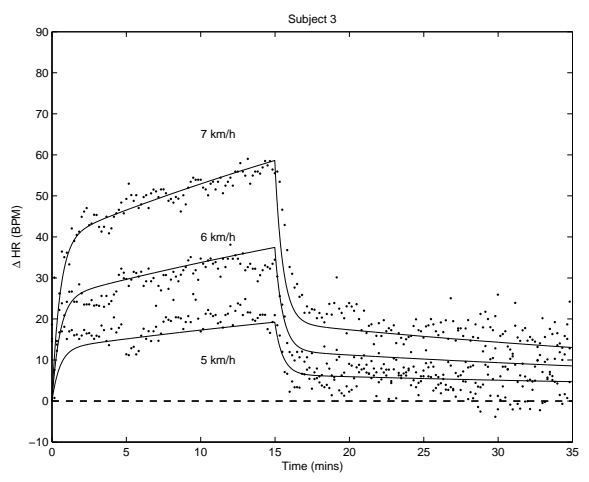

Fig. 3. Heart rate responses of Subject 3: actual responses (dots), simulated responses (solid lines)

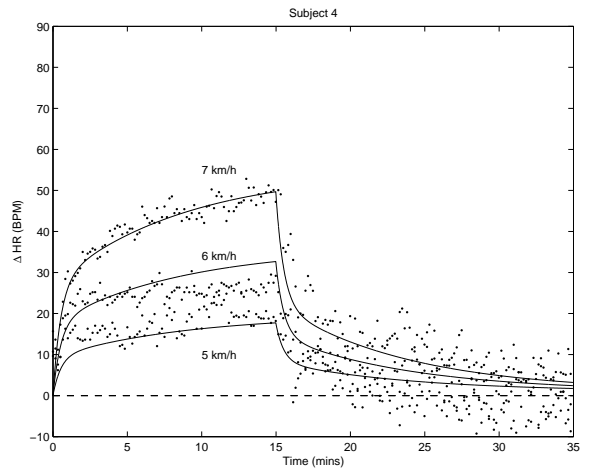

Fig. 4. Heart rate responses of Subject 4: actual responses (dots), simulated responses (solid lines)

The value of $a_{1}$ should decrease when the current heart rate $x_{1}$ is beyond a certain threshold. The drop in $a_{1}$ is due to the switching of the effects from the vagal withdrawal to the sympathetic activation. One remedy to this issue is to consider combining the proposed model with the one in the reference [5].

Nevertheless, there is a great advantage in expressing the heart rate response in the form of the proposed system (1). It is not complicated from the control application point of view. Since system (1) can be written as an uncertain linear control system with an input nonlinearity, techniques like robust Kalman filter [15] and robust $H_{\infty}$ control [16], [17] can be applied for the estimation and control of the system, respectively. More details on the implementation of control and regulation of heart rate can be found in the reference [12].

Since the models were identified by using the heart rate responses to step inputs, it would be important to validate the models by predicting and comparing the heart rate responses to different exercising conditions. This is being carried out in the current research.

\section{ACKNOWLEDGEMENTS}

The authors acknowledge the helpful discussions with Dr Socrates Dokos and Gregory Chan on the topics of parameter estimation and cardiovascular systems, respectively.

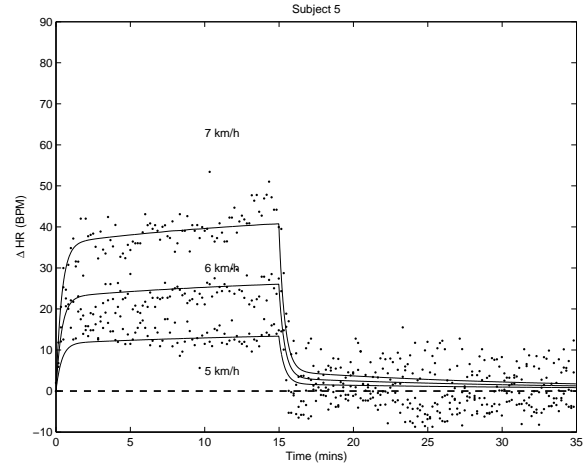

Fig. 5. Heart rate responses of Subject 5: actual responses (dots), simulated responses (solid lines)

\section{REFERENCES}

[1] J. Achten and A. E. Jeukendrup, "Heart rate monitoring: Applications and limitations," Sports Med., vol. 33, no. 7, pp. 517-538, 2003.

[2] K. P. Savonen, T. A. Laka, J. A. Laukkanen, P. M. Halonen, T. H. Rauramaa, J. T. Salonen, and R. Rauramaa, "Heart rate response during exercise test and cardiovascular mortality in middle-aged men," European Heart Journal, vol. 27, pp. 582-588, 2006.

[3] C. R. Cole, E. H. Blackstone, C. E. S. F. J. Pashkow, and M. S. Lauer, "Heart rate recovery immediately after exercise as a predictor of mortality," The New England Journal of Medicine, vol. 341, no. 18 , pp. 1351-1357, 1999.

[4] V. Brodan, M. Hajek, and E. Kuhn, "An analog model of pulse rate during physical load and recovery," Physiologia Bohemoslovaca, vol. 20, pp. 189-198, 1971.

[5] M. Hajek, J. Potucek, and V. Brodan, "Mathematical model of heart rate regulation during exercise," Automatica, vol. 16, pp. 191-195, 1980.

[6] L. B. Rowell, Human Cardiovascular Control. New York: Oxford University Press, 1993.

[7] J. M. Johnson and L. B. Rowell, "Forearm and skin vascular responses to prolonged exercise in man," Journal of Applied Physiology, vol. 39, pp. $920-924,1975$

[8] E. F. Coyle and G. Alonso, "Cardiovascular drift during prolonged exercise: New perspectives," Exercise and Sports Science Review, vol. 29 , pp. 88-92, 2001

[9] R. L. Hughson, "Regulation of blood flow at the onset of exercise by feed forward and feedback mechanisms," Can. J. Appl. Physiol., vol. 28, no. 5, pp. 774-787, 2003.

[10] W. D. McArdle, F. I. Katch, and V. L. Katch, Exercise Physiology: Energy, Nutrition \& Human Performance, 6th ed. Philadelphia, PA: Lippincott Williams \& Wilkins, 2007.

[11] P. E. Martin and D. J. Sanderson, "Biomechanics of walking and running," in Exercise and Sport Science, W. E. Garrett and D. T. Kirkendall, Eds. Philadelphia, PA: Lippincott Williams \& Wilkins, 2000 , pp. 661-674.

[12] S. W. Su, L. Wang, B. G. Celler, A. V. Savkin, and Y. Guo, "Identification and control for heart rate regulation during treadmill exercise," IEEE Transactions on Biomedical Engineering, vol. 54, no. 7, pp. 1238-1246, 2007.

[13] P. Englezos and N. Kalogerakis, Applied Parameter Estimation for Chemical Engineers. New York: Marcel Dekker, 2001.

[14] W. J. H. Stortelder, "Parameter estimation in dynamic systems," Mathematics and Computers in Simulation, vol. 42, pp. 135-142, 1996.

[15] I. R. Petersen and A. V. Savkin, Robust Kalman Filtering for Signals and Systems with Large Uncertainties. Boston: Birkhäuser, 1999.

[16] I. R. Petersen, V. A. Ugrinovskii, and A. V. Savkin, Robust Control Design Using $H^{\infty}$ Methods. London: Springer-Verlag, 2000.

[17] A. V. Savkin and I. R. Petersen, "Nonlinear versus linear control in the robust stabilizability of linear uncertain systems via fixed-order output feedback," IEEE Trans. Automat. Contr., vol. 41, no. 9, pp. $1335-1338,1996$ 